\title{
Application of the Rayleigh-Ritz variational technique for coronal loop oscillations
}

\author{
Narges Fathalian ${ }^{1}$, Hossein Safari ${ }^{2}$ and Sadollah Nasiri ${ }^{1,2}$ \\ ${ }^{1}$ Institute for Advanced Studies in Basic Sciences, P. O. Box 45195-1159, Zanjan, Iran \\ ${ }^{2}$ Department of Physics, Zanjan University, P. O. Box 45195-313, Zanjan, Iran
}

\begin{abstract}
We studied the fast kink modes of a cylindrical model of coronal loops, in coronal conditions, stratified density and low- $\beta$ plasma. The mode frequencies and profiles are calculated.
\end{abstract}

Keywords. Sun: corona, Sun:oscillations

\section{Introduction}

Several theoretical models have been developed to explore the nature and propagation of waves of coronal loops in 1-D models. Here, a Rayleigh-Ritz variational method developed for the oscillations of 2-D coronal loop model.

\section{Overview}

The MHD equations in the matrix representation reduces as (see e.g., Sobouti 1981 and Hasan \& Sobouti 1987).

$$
\mathbf{W Z}=\mathbf{S Z E}
$$

where $\mathrm{E}$ is a diagonal matrix whose elements are the eigenvalues $\omega_{l}$ and $\mathbf{Z}$ is the matrix of the expansion coefficients. The elements of $\mathbf{W}$ and $\mathbf{S}$ are determined from the equations of motions. We adapt a Rayleigh-Ritz procedure and approximate the linear series in Eq. (2.1) by a finite number of terms, say $n$. The matrix blocks $S_{l s}$ and $W_{l s} ; l, s=1,2,3$ become $n \times n$ matrices.

\section{Results}

Our numerical results show that: a) for unstratified loop, as expected, $\omega_{n}$ is proportional to its mode number (i.e., $\left.\omega_{n} \approx n \omega_{1}\right)$. b) for stratified loops, all the fundamental, first, second, and the third overtone kink $(m=1)$ frequencies increases with increasing stratified density scale height. For typical loop lengths, $100-400 \mathrm{Mm}$, the density scale heights fall in the range of $13-108 \mathrm{Mm}$. These results are in agreement with Verwichte et al. (2004), McEwan et al. (2006), and Safari et al. (2007). The application of variational method in real model of coronal loop are implementing in our group.

\section{References}

Hasan, S. S. \& Sobouti, Y. 1987, MNRAS, 228, 427

McEwan, M., Donnelly, G. R., Díaz, A. J., \& Roberts, B. 2006, A\&A, 460, 893

Safari, H., Nasiri, S., \& Sobouti Y. 2007, $A \& A$, 470, 1111

Sobouti, Y. 1981, A\&A, 100, 319

Verwichte, E., Nakariakov, V. M., Ofman, L., \& DeLuca, E. E. 2004, Sol. Phys., 223, 77 